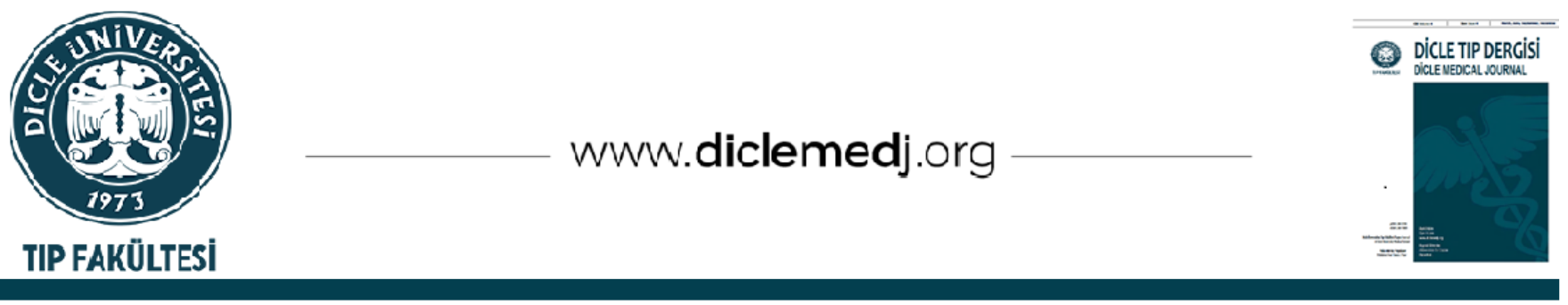

\title{
Evaluation of caregiver burden and quality of life in metastatic prostate cancer patients; Effects of clinical and socioeconomic factors
}

\author{
Ozgen Ahmet Yildirim ${ }^{1}$, Kerem Poyraz ${ }^{i}{ }^{2}$, Erkan Erdur ${ }^{1}{ }^{1}$, Canan can ${ }^{1}$, Cihan Gundogan \\ 3, Halis Yerlikay 4, Serdar Yildiri. 5, Yunus Guz iD 3, Halil Köme 3 \\ 1 Department of Internal Medicine, Division of Medical Oncology, Gazi Yasargil Training and Research Hospital, Diyarbakir, Turkey \\ 2 Department of Radiation Oncology, Gazi Yasargil Training and Research Hospital, Diyarbakir, Turkey
}

3 Department of Nuclear Medicine, Gazi Yasargil Training and Research Hospital, Diyarbakir, Turkey

4 Department of Medical Oncology, Memorial Diyarbakir Hospital, Diyarbakir, Turkey

5 Department of Internal Medicine, Gazi Yasargil Training and Research Hospital, Diyarbakir, Turkey

Received: 16.10.2021; Revised: 27.11.2021; Accepted: 29.11.2021

\begin{abstract}
Objective: To investigate the caregiver burden and quality of life (QoL) of patients with metastatic prostate cancer and, to evaluate the relationship between questionnaire results and medical, socioeconomic, disease and treatment related factors of the patients.

Methods: One hundred twenty one metastatic prostate cancer patients, and caregivers were enrolled in this cross-sectional study. Patients with metastatic prostate cancer who had at least one bone, lymph node or visceral metastasis who had undergone androgen deprivation or chemotherapy treatments for at least 3 months were included. Short Form 36 (SF-36) questionnaires and Zarit caregiver burden interviews (ZCBI) were conducted with patients and their caregivers seperately. Patients' disease, treatment, socioeconomic and caregiver related factors were recorded respectively.

Results: Patients' comorbidities, ECOG score, presence and quantity of bone or visceral metastasis, bone related events, receiving palliative radiotherapy and chemotherapy, using public transportation resulted in lower SF-36 and higher ZCBI results ( $<<0.001-$ $\mathrm{p}=0.049$ ). Obligation to live in rented houses were correlated with lower SF-36 physical function results ( $p=0.043$ ). Condition of the caregiver being from distant relatives resulted in a lower SF-36 score $(\mathrm{p}=0.01-\mathrm{p}=0.043)$. Moderate to strong negative correlation was detected between ZCBI and all SF-36 results ( $\mathrm{p}<0.001$, r:-646 to-749). In ZCBI, score over 47 resulted with $69.57 \%$ sensitivity and $65.52 \%$ specificity (AUC $=0.671, \mathrm{p}=0.008$ ).

Conclusion: QoL was directly related to caregiver burden. Factors such as patient comorbidities, bone metastasis and related situations, ECOG score, presence of visceral metastasis, undergoing chemotherapy, low socioeconomic status negatively affect the quality of life of patients and caregiver burden. If these factors can be monitored, the care and quality of life of these patients can be improved.
\end{abstract}

Keywords: Caregiver burden, quality of life, prostate cancer

DOI: 10.5798/dicletip.1037634

Correspondence / Yazışma Adresi: Ozgen Ahmet Yildirim, Gazi Yasargil Training and Research Hospital Department of Internal Medicine, Division of Medical Oncology Diyarbakir, Turkey, 21070 e-mail: ozgenayildirim@gmail.com 


\section{Metastatik prostat kanserli hastalarda bakıcı yükü ve yașam kalitesinin değerlendirilmesi; Klinik ve sosyoekonomik faktörlerin etkileri}

Öz

Amaç: Metastatik prostat kanserli hastaların bakım veren yükünü ve yaşam kalitesini (QoL) araştırmak ve anket sonuçları ile hastaların tıbbi, sosyoekonomik, hastalık ve tedavi ile ilgili faktörleri arasındaki ilişkiyi değerlendirmek.

Yöntemler: Bu kesitsel çalışmaya yüz yirmi bir metastatik prostat kanserli hasta ve bakım verenler dahil edildi. En az bir kemik, lenf nodu veya viseral metastazı olan, en az 3 aydır androjen deprivasyon veya kemoterapi tedavisi gören metastatik prostat kanserli hastalar dahil edildi. Hastalar ve bakım verenleri ile ayrı ayrı Kısa Form 36 (SF-36) anketleri ve Zarit bakıcı yükü görüșmeleri (ZCBI) yapılmıştır. Hastaların hastalığı, tedavisi, sosyoekonomik ve bakım verenle ilgili faktörler sırasıyla kaydedildi.

Bulgular: Hastaların komorbiditeleri, ECOG skoru, kemik veya viseral metastaz varlığı ve miktarı, kemikle ilgili olaylar, palyatif radyoterapi ve kemoterapi almaları, toplu taşıma kullanmaları SF-36'nın daha düşük ve ZCBI sonuçlarının daha yüksek olmasına neden oldu $(\mathrm{p}<0,001-\mathrm{p}=0,049)$. Kiralık evlerde yaşama zorunluluğu, daha düşük SF-36 fiziksel fonksiyon sonuçları ile korele idi ( $p=0.043$ ). Bakım verenin uzak akrabadan olması, KF-36 puanının daha düşük olmasına neden oldu ( $p=0.01-p=0.043)$. ZCBI ve tüm SF-36 sonuçları arasında orta ila güçlü negatif korelasyon saptandı ( $p<0,001, r$ :-646 ila-749). ZCBI'da 47'nin üzerindeki puan, \%69,57 duyarlılık ve \%65.52 özgüllük ile sonuçlandı (AUC =0.671, p =0.008).

Sonuç: Yaşam kalitesi, bakım verenin yükü ile doğrudan ilişkilidir. Hasta komorbiditeleri, kemik metastazı ve ilişkili durumlar, ECOG skoru, viseral metastaz varlığı, kemoterapi uygulanması, düşük sosyoekonomik durum gibi faktörler hastaların yaşam kalitesini ve bakım verenin yükünü olumsuz etkilemektedir. Bu faktörlerin monitorizasyonu sağlanabilir ise, bu hastaların bakımı ve yaşam kaliteleri artırılabilir.

Anahtar kelimeler: Bakıcı yükü, yaşam kalitesi, prostat kanseri.

\section{INTRODUCTION}

Patients and caregivers have some concerns about reducing daily productivity or missed business days due to hospitality and long- term complications of treatment or disease itself. The idea of having a potential or absolute mortal disease, sustained therapies and disease course could be devastating on feelings in the individuals and family members ${ }^{1}$. Therefore, caregivers of cancer patients might feel burden, and as a result patients might seek for poor quality of life conditions. Caregiver burden and quality of life (QoL) have been investigated in various diseases such as chronic kidney disease including patients receiving dialysis and transplant recipients, schizophrenia, Parkinson's disease, inflammatory bowel disease and Alzheimer's disease ${ }^{2-6}$.

There is limited knowledge in the literature regarding caregiver burden with QoL in cancer. Johansen $\mathrm{S}$ et al. were determined the factors that effects caregiver burden of cancer patients. There is a relationship between the patient factors such as sleep disturbance, fatigue and cancer symptoms with caregiver burden. Additionally caregiver's factors such as being female, having sleep disturbance and other chronic illnesses significantly impacts the burden ${ }^{7}$. In advanced cancer, the severe patient's physical and mental health is associated with a heavier caregiver burden, regardless of caregiving hours ${ }^{8}$. Crowder et al. have been determined that head and neck cancer patients with a long illness age adapt to daily life restrictions. Most of these patients continue to have unmet needs. They experience a period of accepting that some of their needs are not met in the coping process, and this situation is associated with depressive status of the patients ${ }^{9}$. Guan $\mathrm{T}$ et al. found that uncertainty in the course of the disease was associated with mental well-being disorders ${ }^{10}$.

There are several studies evaluating the quality of life of prostate cancer patients. However, there is no study in the literature evaluating QoL and burden in prostate cancers, our study is important in this context and will be the first study in the literature. The survival and quality 
of life of patients have increased with new generation anti-androgen treatments. A recent meta-analysis revealed that apalutamide and abiraterone acetate provided the best overall survival and enzalutamide provided the best radiological progression-free survival ${ }^{11}$. With advancing treatments, prostate cancer patients who have a long disease duration and can reach older ages represent an important segment in the patient profile. In the first 24 months of the disease, although tolerance of all treatment modalities is good, each has a different effect on the quality of life ${ }^{12}$.

In this study, we aimed to investigate the caregiver burden and quality of life of patients with metastatic prostate cancer. With the results we hoped to achieve, our aim was to provide a systematic quality of life analysis and caregiver burden monitoring. We started our study with the foresight that it can contribute to the formation of a guide in terms of rational and effective distribution of healthcare services whose economic burden is growing day by day.

\section{METHODS}

Design, Setting and Patients: One hundred twenty one metastatic prostate cancer patients and primary caregivers were enrolled in this descriptive cross-sectional study from October 2019 to October 2020. Patients aged 50-91 years who have had the illness for three months or more, followed-up in Gazi Yasargil Training and Research Hospital. Additionally, the primary caregivers of the patients who gave consent for participation were enrolled in the study. All patients had histopathological verification for prostate cancer diagnosis. The diagnosis of metastatic prostate cancer was confirmed with Galium-68 prostate specific membrane antigen positron emulsion tomography.

All patients and their primary caregivers (spouse or children) were evaluated in the same meeting room at the Medical Oncology
Department of Gazi Yasargil Training and Research Hospital. The evaluation was performed by a medical oncology specialist.

Inclusion Criteria: Patients with metastatic prostate cancer who had at least one bone, lymph node or visceral metastasis who had undergone androgen deprivation or chemotherapy treatments for at least 3 months were included.

Exclusion Criteria: Patients who had an additional uncontrolled chronic disease such as severe hypertension, dis-regulated diabetes mellitus, any hearth disease, any type of ischemic cerebrovascular disease and any type of baseline orthopedic failure that causes mobilization defect, were excluded from the study. The exclusion criterion for hypertension was blood pressure above 170/100 mm / hg in at least 2 consecutive measurements despite using antihypertensive drugs. Diabetic patients with an HbA1c level above 7.5 or using insulin therapy or using more than one oral antidiabetic agents were excluded. Patients with an HbA1c level below 7.5 with metformin treatment or single oral anti-diabetic therapy were included in the study. The exclusion criteria of diabetic patients were evaluated according to the relevant age group recommendations of the American diabetes association $^{13}$.

Patients who receive second-generation androgen pathway inhibitors were not included in our study.

Data Collection and Questionnaires: Two selfadministered surveys were completed by caregivers as follows:

Short Form 36 (SF-36): This questionnaire was applied to patient's caregivers. McHorney et al performed analysis of cross-sectional data, physical and mental health status with 36-item short form questionnaire. Survey includes a comparison with traditional psychometric and clinical tests. Principal component analysis 
method was used for the assumed physical and mental health analysis. Physical health analysis includes assessments stratified by the severity of the chronic medical condition while measuring physical function and physical role limitations. It also evaluates role limitations and emotional status in terms of mental health ${ }^{14}$. SF36 questionnaire contains sub-results such as physical function (PF), physical role (PR), bodily pain (BP), general health $(\mathrm{GH})$, vitality (V), social functioning (SF), emotional role (ER) and mental health (M).

ZCBI Caregiver Burden Interview (ZCBI): This questionnaire was applied to caregivers whom were patients' relatives. ZCBI was created by Zarit et al. ${ }^{15}$ in 1980 as a caregiver self-report measure. The revised version of this scale consists of 22 items about physical health, psychological well-being, financial status and interpersonal relationships to evaluate caregiver burden and the impact of the patient's disease on a caregiver's life ${ }^{15}$. Ozlu et al.16 developed the Turkish-validated ZCBI. On investigating the validity and reliability of the Turkish-language version, three items (numbers 1, 4 and 16) were removed. Hence, the Turkish-language version consists of 19 items. The scale was developed to be selfadministered; however, it can also be administered by an interviewer. In the present study, the interviewer read each item aloud and asked the respondent to state their answer. Each item is scored from 1 to $5,1=$ never, $2=$ rarely, 3 = sometimes, 4 = quite frequently, and 5 = nearly always. Caregiver burden is evaluated on the basis of the total score obtained from the sum of the responses. The total scores were calculated to be between 19 and 95 points, and higher scores indicating higher caregiver burden. A score of 21 or less indicates no burden, 22-46 indicates mild burden, 47-55 means moderate burden and 56 or more indicates severe burden.

\section{Stratification Factors}

Patient related clinical factors: Age, comorbidities, ECOG score, presence of bone metastasis, presence of bone-related event and organ metastasis were determined. The bonerelated event was defined as the need for opioid use due to severe bone pain or as an incurred bone fracture.The number of bone metastases was also stratified as 0, 1-3 and >4 metastases. The reason for this stratification method was that the cut off value for the distinction between oligometastatic disease and diffuse metastatic disease had been generally considered to be between 3 and 5 metastases by most authors ${ }^{17,18 .}$

Treatment related factors: All patients received androgen deprivation therapy. The treatment related factors were chemotherapy which was accepted as active receiving in terms of cross sectional data collecting. History of chemotherapy within six months was included as active chemotherapy too. In addition, whether the patients received palliative radiotherapy, prostatic region radiotherapy and prostatectomy was also planned to examine.

Economic and educational factors: Patient educational status, personal vehicle presence or public transport usage of family, place of residence (city center, village), residence status (own house - rent) were determined

Caregiver-related factors: Caregiver age, the degree of proximity of caregivers such as spouse, child, sibling or other relatives, caregiver education and caregiver comorbidities were identified as caregiverrelated factors.

\section{Statistical Analysis and Ethics}

SPSS (Statistical Package for the Social Sciences) 23.0 package program was used for the statistical analysis of the data. Categorical measurements were summarized as numbers and percentages, and continuous measurements as mean and standard deviation (median and minimum- 
maximum where necessary). The suitability of the variables to normal distribution was examined using the Shapiro-Wilk Tests. Mann Whitney U test was used in paired groups and Kruskall Wallis tests were used in more than two groups for parameters that did not show normal distribution. Bonferroni method, one of the Post Hoc tests, was used to determine the source of the difference between the groups. While multiple regression model was used to determine the relationship between Zarit score and other parameters, Spearman Correlation analysis was used to determine the relationship between Zarit score and age, caregiver age and SF36 scores. The statistical significance level was taken as 0.05 in all tests.

This study was approved by The Gazi Yaşargil Training and Research Hospital Ethics Board (25.09.2020/564) and was applied in accordance of the Declaration of Helsinki. Informed consents were obtained from each participants and caregivers.

\section{RESULTS}

Median 71 years old (51-92) 121 prostate cancer patients included to our study. Evaluated demographic results and frequencies are given in the first columns of tables 1 and 2 . When we looked at the patient-related factors affecting the ZCBI and SF-36 results, we found that the presence of comorbidity had a statistically significant effect on the SF-36 results and the ZCBI results $(p<0.001)$. Low SF-36 and high ZCBI results were observed with the increase in ECOG performance score ( $p<0.001)$. In post-hoc analysis, it was observed that every 1 unit increase of ECOG made a significant difference in these results ( $p<0.001$ - 0.007). While SF-36 results were affected by the presence of bone metastasis $(p=0.001-p=0.036)$, it was observed that this factor did not significantly affect the results of ZCBI. Regarding the examination in terms of the number of bone metastases; patients with no metastases, 1-3 metastases and $>4$ metastases were evaluated comparatively. Posthoc analysis showed that the number of bone metastases affected all questionnaire results $(p$ $<0.001-p=0.013)$. When we look at the anamnesis in terms of complications due to bone metastasis, it was observed that both SF-36 and ZCBI questionnaire results were worse in cases with complications ( $p<0.001-p=0.044)$. The presence of visceral metastasis was similarly associated with low SF-36 results and high ZCBI caregiver burden $(p<0.001-p=0.006)$ (table 1$)$.

When treatment related factors were considered; prostatectomy and radiotherapy to the prostatic region did not appear to have a statistically significant effect on the survey results. However, it was observed that SF-36 results in PF, PR, BP, $\mathrm{SF}, \mathrm{ER}$ and $\mathrm{M}$ results were negatively affected in patients who were actively receiving chemotherapy $(\mathrm{p}<0.001-\mathrm{p}=0.049)$. Patients who received palliative radiotherapy had lower SF-36 and higher ZCBI results $(p<0.01-p=0.012)$ (table 1).

When we looked at the economic and educational factors, we observed that the educational status did not have a direct effect on the quality of life from illiterate to university graduates. It was observed that those with private means of transport had statistically significantly higher SF36 and lower ZCBI caregiver burden results than those who had to use public transport ( $p=0.009$ $p=0.048$ ). However, it was also observed that the difference in SF-36 GH, SF and M results did not reach statistical significance (table 2).

In order to investigate the degree of kinship related to the caregiver; the situations of the caregiver being spouse, children, siblings and other relatives were compared. In SF-36 PF, BP and $M$ results, it was observed that the condition of the caregiver being from distant relatives constituted a lower quality of life score $(p=0.01$ $p=0.043$ ). It was observed that the situation of siblings being the caregivers were associated with better SF-36 BP and GH results than children as caregivers ( $p=0.025-0.026)$. It was observed that whether the caregiver is female or male did not make a statistically significant difference ( $\mathrm{p}>$ 0.1 ). It was also observed that caregiver's own comorbidities presence or absence did not significantly affect the results ( $p>0.07)$ (table 2$)$. 
Table I: Patient and treatment related factors affecting Zarit and Short Form 36 questionnaires

\begin{tabular}{|c|c|c|c|c|c|c|c|c|c|}
\hline & $\begin{array}{r}\text { SF36-PF } \\
\text { Med (Min- } \\
\text { Max) }\end{array}$ & $\begin{array}{r}\text { SF36-PR } \\
\text { Med (Min- } \\
\text { Max) }\end{array}$ & $\begin{array}{r}\text { SF36-BP } \\
\text { Med (Min- } \\
\text { Max) }\end{array}$ & $\begin{array}{r}\text { SF36-GH } \\
\text { Med (Min- } \\
\text { Max) }\end{array}$ & $\begin{array}{r}\text { SF36-V } \\
\text { Med (Min- } \\
\text { Max) }\end{array}$ & $\begin{array}{r}\text { SF36-SF } \\
\text { Med (Min- } \\
\text { Max) }\end{array}$ & $\begin{array}{r}\text { SF36-ER } \\
\text { Med (Min- } \\
\text { Max) }\end{array}$ & $\begin{array}{r}\text { SF36-M } \\
\text { Med (Min- } \\
\text { Max) }\end{array}$ & $\begin{array}{r}\text { Zarit } \\
\text { Med (Min- } \\
\text { Max) }\end{array}$ \\
\hline \multicolumn{10}{|l|}{ Comorbidity } \\
\hline $\begin{array}{l}\text { Absent } \\
n=21(17.4 \%)\end{array}$ & $63(27-71)$ & $60(22-66)$ & $60(32-62)$ & $61(33-68)$ & $52(33-56)$ & $63(33-78)$ & $60(26-66)$ & $60(34-68)$ & $39(24-63)$ \\
\hline $\begin{array}{l}\text { Present } \\
n=100(82.6 \%)\end{array}$ & $33(18-65)$ & $30(12-64)$ & $40(24-64)$ & $40(24-66)$ & $40(24-63)$ & $50(24-67)$ & $31(20-66)$ & $52(24-64)$ & $55(26-79)$ \\
\hline p value & $<0,001$ & $<0,001$ & $<0,001$ & $<0,001$ & 0,001 & $<0,001$ & $<0,001$ & $<0,001$ & $<0,001$ \\
\hline \multicolumn{10}{|l|}{ ECOG score } \\
\hline $\begin{array}{l}1 \\
n=14(11.6 \%)\end{array}$ & $66(24-71)$ & $62(29-66)$ & $60(26-62)$ & $\begin{array}{l}63,5 \quad(25- \\
68)\end{array}$ & $53(24-56)$ & $\begin{array}{r}66,5(26- \\
78)\end{array}$ & $\begin{array}{r}62,5(28- \\
66)\end{array}$ & $61(26-68)$ & $\begin{array}{r}30,5(24- \\
77)\end{array}$ \\
\hline $\begin{array}{l}2 \\
n=87(71.9 \%)\end{array}$ & $35(24-64)$ & $32(23-64)$ & $40(24-64)$ & $40(24-66)$ & $40(24-63)$ & $50(24-66)$ & $34(24-66)$ & $56(24-64)$ & $50(26-77)$ \\
\hline $\begin{array}{l}3 \\
n=20\end{array}$ & $24,5(18-27)$ & $\begin{array}{r}20,5(12- \\
22)\end{array}$ & $40(36-43)$ & $39(33-43)$ & $39(33-43)$ & $46(32-52)$ & $23(20-33)$ & $\begin{array}{r}49,5(35- \\
59)\end{array}$ & $61(41-79)$ \\
\hline p value & $<0,001$ & $<0,001$ & $<0,001$ & $<0,001$ & $<0,001$ & $<0,001$ & $<0,001$ & $<0,001$ & $<0,001$ \\
\hline \multicolumn{10}{|l|}{ Bone met. } \\
\hline $\begin{array}{l}\text { Absent } \\
\mathrm{n}=6 \text { (5\%) }\end{array}$ & $60(29-64)$ & $62(24-64)$ & $\begin{array}{r}61,5(40- \\
64)\end{array}$ & $\begin{array}{r}62,5(40- \\
66)\end{array}$ & $\begin{array}{r}61,5(38- \\
63)\end{array}$ & $\begin{array}{r}63,5(49- \\
66)\end{array}$ & $\begin{array}{r}63,5(25- \\
66)\end{array}$ & $60(59-64)$ & $40(33-54)$ \\
\hline $\begin{array}{l}\text { Present } \\
n=115(95 \%)\end{array}$ & 34 (18-71) & $31(12-66)$ & $40(24-62)$ & 40 (24-68) & $40(24-56)$ & $50(24-78)$ & $32(20-66)$ & $53(24-68)$ & $51(24-79)$ \\
\hline p value & 0,032 & 0,008 & 0,001 & 0,004 & 0,002 & 0,036 & 0,005 & 0,004 & 0,057 \\
\hline \multicolumn{10}{|l|}{ BM quantity } \\
\hline $\begin{array}{l}0 \\
n=6(5 \%)\end{array}$ & $60(29-64)$ & $62(24-64)$ & $\begin{array}{r}61,5(40- \\
64)\end{array}$ & $\begin{array}{r}62,5(40- \\
66)\end{array}$ & $\begin{array}{r}61,5(38- \\
63)\end{array}$ & $\begin{array}{r}63,5(49- \\
66)\end{array}$ & $\begin{array}{r}63,5(25- \\
66)\end{array}$ & $66(59-64)$ & $40(33-54)$ \\
\hline $\begin{array}{l}1-3 \\
n=33(27.3 \%)\end{array}$ & $42(28-71)$ & $39(23-66)$ & $50(28-62)$ & $52(28-68)$ & $43(28-56)$ & $55(28-78)$ & $43(24-66)$ & $59(24-68)$ & 45 (24-77) \\
\hline $\begin{array}{l}4 \text { or more } \\
n=82(67.8 \%)\end{array}$ & $32(18-67)$ & $\begin{array}{r}29,5(12- \\
63)\end{array}$ & $40(24-62)$ & $40(24-64)$ & 39 (24-56) & 49 (24-68) & $30(20-63)$ & $51(24-62)$ & $56(26-79)$ \\
\hline$p$ value & $<0,001$ & $<0,001$ & $<0,001$ & $<0,001$ & $<0,001$ & $<0,001$ & $<0,001$ & $<0,001$ & $<0,001$ \\
\hline \multicolumn{10}{|l|}{ Bone event } \\
\hline \multirow{3}{*}{$\begin{array}{l}\text { Absent } \\
n=95(78.5 \%) \\
\text { Present } \\
n=26(21.5 \%) \\
\text { p value }\end{array}$} & $37(20-71)$ & $32(12-66)$ & $42(24-64)$ & $43(24-68)$ & $41(24-63)$ & $52(24-78)$ & $32(20-66)$ & $59(24-68)$ & $48(24-77)$ \\
\hline & $32(18-42)$ & $30(12-39)$ & $33(26-43)$ & $36(25-42)$ & 37 (24-42) & $\begin{array}{r}34,5(26- \\
44)\end{array}$ & $33(22-48)$ & $36(26-40)$ & $\begin{array}{r}58,5 \text { (51- } \\
79)\end{array}$ \\
\hline & 0,001 & 0,025 & $<0,001$ & $<0,001$ & $<0,001$ & $<0,001$ & 0,114 & $<0,001$ & $<0,001$ \\
\hline \multicolumn{10}{|l|}{ Visceral met. } \\
\hline $\begin{array}{l}\text { Absent } \\
n=112 \text { (92.6\%) }\end{array}$ & $35,5(18-71)$ & $32(12-66)$ & $41(30-64)$ & $41(30-68)$ & $40(33-63)$ & $51(32-78)$ & $34(20-66)$ & $\begin{array}{r}56,5(32- \\
68)\end{array}$ & $\begin{array}{r}49,5 \text { (24- } \\
79)\end{array}$ \\
\hline $\begin{array}{l}\text { Present } \\
\mathrm{n}=9(7.4 \%)\end{array}$ & 27 (24-33) & $26(23-38)$ & $26(24-29)$ & 27 (24-29) & $26(24-28)$ & $26(24-28)$ & 27 (24-28) & $26(24-29)$ & $67(60-77)$ \\
\hline p value & 0,002 & 0,044 & $<0,001$ & $<0,001$ & $<0,001$ & $<0,001$ & 0,006 & $<0,001$ & $<0,001$ \\
\hline \multicolumn{10}{|l|}{ Prostatectomy } \\
\hline \multirow{3}{*}{$\begin{array}{l}\text { Absent } \\
n=105(86.8 \%) \\
\text { Present } \\
n=16(13.2 \%) \\
\text { p value }\end{array}$} & $34(18-71)$ & $32(12-66)$ & $41(24-63)$ & $40(24-68)$ & $40(24-63)$ & $50(24-78)$ & $32(20-66)$ & $56(24-68)$ & $51(24-79)$ \\
\hline & $36,5(26-66)$ & $32(21-64)$ & $40(27-64)$ & $\begin{array}{r}40,5(29- \\
66)\end{array}$ & $\begin{array}{r}39,5(27- \\
61)\end{array}$ & $50(28-67)$ & $\begin{array}{r}34,5(24- \\
66)\end{array}$ & $\begin{array}{r}51,5(29- \\
62)\end{array}$ & $\begin{array}{r}53,5(26- \\
69)\end{array}$ \\
\hline & 0,469 & 0,632 & 0,869 & 0,960 & 0,431 & 0,976 & 0,579 & 0,560 & 0,668 \\
\hline \multicolumn{10}{|l|}{ Pr.RT } \\
\hline \multirow{2}{*}{$\begin{array}{l}\text { Absent } \\
n=112(92.6 \%) \\
\text { Present } \\
n=9(7.4 \%)\end{array}$} & $34(18-71)$ & $\begin{array}{r}31,5(12- \\
66)\end{array}$ & $\begin{array}{r}40,5(24- \\
64)\end{array}$ & 40 (24-68) & $40(24-63)$ & $50(24-78)$ & $\begin{array}{r}32,5(20- \\
66)\end{array}$ & $55(24-68)$ & $51(24-79)$ \\
\hline & $35(28-64)$ & $33(23-60)$ & $40(30-61)$ & $42(32-62)$ & $41(39-55)$ & $53(36-64)$ & $35(24-60)$ & $57(35-62)$ & $47(42-67)$ \\
\hline
\end{tabular}




\begin{tabular}{|c|c|c|c|c|c|c|c|c|c|}
\hline p value & 0,474 & 0,649 & 0,855 & 0,461 & 0,229 & 0,315 & 0,711 & 0,536 & 0,965 \\
\hline \multicolumn{10}{|l|}{ Chemotherapy } \\
\hline $\begin{array}{l}\text { Absent } \\
n=68(56.2 \%)\end{array}$ & $32(18-67)$ & $29(12-64)$ & $40(25-62)$ & $40(25-64)$ & $40(24-60)$ & $50(24-68)$ & $\begin{array}{r}29,5(20- \\
66)\end{array}$ & $52(24-64)$ & $\begin{array}{r}56,5(24- \\
79)\end{array}$ \\
\hline $\begin{array}{l}\text { Present } \\
n=53(43.8 \%)\end{array}$ & $40(23-71)$ & $38(20-66)$ & $43(24-64)$ & $43(24-68)$ & $41(24-63)$ & $52(24-78)$ & $38(22-66)$ & $59(24-68)$ & $48(24-77)$ \\
\hline p value & $<0,001$ & $<0,001$ & 0,020 & 0,078 & 0,121 & 0,049 & $<0,001$ & 0,100 & 0,009 \\
\hline \multicolumn{10}{|l|}{ Pal.RT } \\
\hline $\begin{array}{l}\text { Absent } \\
n=88(72.7 \%)\end{array}$ & $37,5(18-71)$ & $\begin{array}{r}33,5(12- \\
66)\end{array}$ & $42(24-64)$ & 43 (24-68) & $41(24-63)$ & $52(24-78)$ & $\begin{array}{r}34,5(22- \\
66)\end{array}$ & $\begin{array}{r}58,5(24- \\
68)\end{array}$ & 48 (24-79) \\
\hline $\begin{array}{l}\text { Present } \\
n=33(27.3 \%)\end{array}$ & $31(20-51)$ & $29(12-48)$ & $36(25-60)$ & $37(25-60)$ & $38(24-53)$ & $37(24-56)$ & $30(20-50)$ & 37 (24-59) & $58(40-77)$ \\
\hline p value & $<0,001$ & 0,002 & $<0,001$ & $<0,001$ & $<0,001$ & $<0,001$ & 0,012 & $<0,001$ & $<0,001$ \\
\hline
\end{tabular}

SF: Short Form, PF: physical functioning, PR: physical role, BP: bodily pain, GH: general health, V: vitality, SF: social functioning, ER: emotional role, M: mental health, BM: bone metastasis, met.: metastasis

Table II: Economic and educational factors associated with Short Form 36 and Zarit Caregiver Burden

\begin{tabular}{|c|c|c|c|c|c|c|c|c|c|}
\hline & SF36-PF & SF36-PR & SF36-BP & SF36-GH & SF36-V & SF36-SF & SF36-ER & SF36-M & Zarit \\
\hline & $\begin{array}{r}\text { Med (Min- } \\
\text { Max) }\end{array}$ & Med (Min-Max) & $\begin{array}{r}\text { Med (Min- } \\
\text { Max) }\end{array}$ & $\begin{array}{r}\text { Med (Min- } \\
\text { Max) }\end{array}$ & $\begin{array}{r}\text { Med (Min- } \\
\text { Max) }\end{array}$ & $\begin{array}{r}\text { Med (Min- } \\
\text { Max) }\end{array}$ & $\begin{array}{r}\text { Med (Min- } \\
\text { Max) }\end{array}$ & $\begin{array}{r}\text { Med (Min- } \\
\text { Max) }\end{array}$ & $\begin{array}{r}\text { Med (Min- } \\
\text { Max) }\end{array}$ \\
\hline \multicolumn{10}{|l|}{ Education } \\
\hline $\begin{array}{l}\text { Illiterate } \\
\mathrm{n}=12(9.9 \%)\end{array}$ & $31(23-66)$ & $29,5(18-64)$ & $\begin{array}{r}38,5(26- \\
62)\end{array}$ & $39(25-64)$ & $38(24-60)$ & 47 (26-66) & $\begin{array}{r}30,5(24- \\
66)\end{array}$ & $51(26-64)$ & 57 (26-77) \\
\hline $\begin{array}{l}\text { Literacy } \\
n=35(28.9 \%)\end{array}$ & $32(18-71)$ & $30(12-64)$ & $40(27-63)$ & $41(27-64)$ & $39(27-62)$ & $51(27-74)$ & $30(20-63)$ & $52(24-64)$ & $50(24-79)$ \\
\hline $\begin{array}{l}\text { Primary } \\
n=47(38.8 \%)\end{array}$ & $35(22-69)$ & $32(14-66)$ & $41(24-64)$ & $40(26-67)$ & $40(26-62)$ & $50(24-77)$ & $34(22-66)$ & 57 (26-66) & $49(26-74)$ \\
\hline $\begin{array}{l}\text { H.S-U } \\
n=27(22.3 \%)\end{array}$ & $36(24-70)$ & 35 (21-65) & $40(25-62)$ & $40(24-68)$ & $41(24-63)$ & $51(24-78)$ & $36(22-66)$ & $52(24-68)$ & $51(24-70)$ \\
\hline p value & 0,503 & 0,572 & 0,588 & 0,728 & 0,540 & 0,290 & 0,510 & 0,588 & 0,620 \\
\hline \multicolumn{10}{|l|}{ Transp. } \\
\hline $\begin{array}{l}\text { Public T. } \\
\mathrm{n}=59(48.8 \%)\end{array}$ & $38(27-69)$ & $34(16-66)$ & $43(29-63)$ & $42(25-67)$ & $42(24-63)$ & $51(26-77)$ & $36(28-64)$ & $58(27-68)$ & $51(28-79)$ \\
\hline $\begin{array}{l}\text { Own } \\
n=62(51.2 \%)\end{array}$ & $34(20-71)$ & $31(12-65)$ & $40(24-64)$ & $40(24-68)$ & $40(24-61)$ & 47 (21-78) & $32(20-66)$ & 55 (24-68) & 50 (24-77) \\
\hline p value & 0,009 & 0,019 & 0,028 & 0,063 & 0,046 & 0,064 & 0,048 & 0,07 & 0,032 \\
\hline \multicolumn{10}{|l|}{ Residence } \\
\hline $\begin{array}{l}\text { Village } \\
\mathrm{n}=43(35.5 \%)\end{array}$ & $39(24-71)$ & $41(22-71)$ & 47 (34-73) & $46(32-67)$ & $44(28-66)$ & $54(34-79)$ & $39(30-64)$ & 57 (24-66) & $51(26-79)$ \\
\hline $\begin{array}{l}\text { Central } \\
\mathrm{n}=78(64.5 \%)\end{array}$ & $35(18-70)$ & $33(12-65)$ & $41(25-64)$ & $41(25-68)$ & $40(24-63)$ & $50(24-78)$ & $35(22-66)$ & $52(24-68)$ & 49 (22-77) \\
\hline p value & 0,046 & 0,013 & 0,004 & 0,016 & 0,021 & 0,045 & 0,090 & 0,061 & 0,04 \\
\hline \multicolumn{10}{|l|}{ Residence } \\
\hline $\begin{array}{l}\text { Rent } \\
\mathrm{n}=45 \text { (37.2\%) }\end{array}$ & $38(24-74)$ & 33 (15-69) & $44(25-69)$ & $40(24-68)$ & $40(24-63)$ & $51(24-78)$ & $34(23-66)$ & $52(24-68)$ & $48(22-77)$ \\
\hline $\begin{array}{l}\text { Own } \\
n=76(62.8 \%)\end{array}$ & $34(18-71)$ & $31(12-66)$ & $40(24-63)$ & $41(25-67)$ & $40(24-62)$ & $50(24-77)$ & $32(20-66)$ & $56(24-66)$ & 54 (24-79) \\
\hline p value & 0,043 & 0,079 & 0,054 & 0,526 & 0,725 & 0,849 & 0,438 & 0,337 & 0,151 \\
\hline \multicolumn{10}{|l|}{ Caregiver } \\
\hline $\begin{array}{l}\text { Spouse } \\
n=71(58.7 \%)\end{array}$ & $34(18-70)$ & $31(12-65)$ & $40(25-64)$ & $40(24-68)$ & $40(24-62)$ & 49 (24-78) & $32(22-66)$ & $56(24-68)$ & $51(24-79)$ \\
\hline $\begin{array}{l}\text { Child } \\
\mathrm{n}=24(19.8 \%)\end{array}$ & 38,5 (26-69) & $35,5(21-66)$ & $42(32-62)$ & $46(33-67)$ & $40(34-63)$ & $\begin{array}{r}50,5(34- \\
77)\end{array}$ & $36(24-64)$ & $\begin{array}{r}57,5(33- \\
66)\end{array}$ & 47 (26-69) \\
\hline $\begin{array}{l}\text { Sibling } \\
\mathrm{n}=15(12.4 \%)\end{array}$ & $31(22-43)$ & $30(14-41)$ & $38(24-50)$ & $38(26-52)$ & $40(26-43)$ & $51(24-56)$ & $30(23-43)$ & $48(26-59)$ & $58(32-70)$ \\
\hline
\end{tabular}




\begin{tabular}{|c|c|c|c|c|c|c|c|c|c|}
\hline $\begin{array}{l}\text { Other relative } \\
\mathrm{n}=11(9.1 \%)\end{array}$ & $42(20-71)$ & $39(12-64)$ & $55(38-62)$ & $49(38-64)$ & $41(34-55)$ & $51(41-74)$ & $40(20-63)$ & $59(38-63)$ & $48(24-79)$ \\
\hline p & 0,034 & 0,100 & 0,005 & 0,015 & 0,480 & 0,192 & 0,084 & 0,027 & 0,061 \\
\hline \multicolumn{10}{|l|}{$\begin{array}{l}\text { Caregiver } \\
\text { Gender }\end{array}$} \\
\hline $\begin{array}{l}\text { Female } \\
\mathrm{n}=86(71.1 \%)\end{array}$ & $33,5(18-71)$ & $31(12-65)$ & $40(25-64)$ & $40(24-68)$ & $40(24-62)$ & $\begin{array}{l}49,5 \quad(24- \\
78)\end{array}$ & $32(20-66)$ & $\begin{array}{r}55,5(24- \\
68)\end{array}$ & $\begin{array}{r}52,5(24- \\
79)\end{array}$ \\
\hline $\begin{array}{l}\text { Male } \\
n=35 \text { (28.9\%) }\end{array}$ & $36(22-69)$ & $33(14-66)$ & $41(24-62)$ & $42(26-67)$ & $40(26-63)$ & 51 (24-77) & $35(22-64)$ & $53(26-66)$ & $48(24-70)$ \\
\hline p & 0,315 & 0,567 & 0,235 & 0,115 & 0,350 & 0,238 & 0,565 & 0,412 & 0,145 \\
\hline \multicolumn{10}{|l|}{$\begin{array}{l}\text { Caregiver } \\
\text { Education }\end{array}$} \\
\hline $\begin{array}{l}\text { Illiterate } \\
\mathrm{n}=7(5.8 \%)\end{array}$ & $30(24-64)$ & $29(23-61)$ & $39(26-62)$ & $39(25-64)$ & $42(24-53)$ & $50(26-66)$ & $28(24-63)$ & $56(26-60)$ & $56(26-77)$ \\
\hline $\begin{array}{l}\text { Literacy only } \\
\mathrm{n}=29(24 \%)\end{array}$ & $36(18-68)$ & $31(12-64)$ & $42(24-63)$ & $40(26-64)$ & 41 (24-67) & 50 (24-67) & $32(22-66)$ & $57(26-64)$ & 48 (24-79) \\
\hline $\begin{array}{l}\text { Primary } \\
n=60(49.6 \%)\end{array}$ & $34(22-71)$ & $31,5(14-66)$ & $41(25-64)$ & $\begin{array}{r}41,5(24- \\
67)\end{array}$ & $\begin{array}{r}51,5(24- \\
77)\end{array}$ & $\begin{array}{r}51,5(24- \\
77)\end{array}$ & $33(22-66)$ & $53(24-66)$ & $50(26-77)$ \\
\hline $\begin{array}{l}\text { H.S-U } \\
n=25(20.7 \%)\end{array}$ & $35(20-70)$ & $33(12-65)$ & $40(29-61)$ & 39 (24-68) & 49 (26-78) & $49(26-78)$ & $35(20-66)$ & $51(28-68)$ & $54(24-77)$ \\
\hline p & 0,842 & 0,985 & 0,730 & 0,728 & 0,370 & 0,480 & 0,972 & 0,597 & 0,954 \\
\hline \multicolumn{10}{|c|}{ Caregiver Comorbidity } \\
\hline $\begin{array}{l}\text { Absent } \\
n=68(56.2 \%)\end{array}$ & $34(18-70)$ & $31(12-66)$ & $40(24-64)$ & $40(24-68)$ & $39(25-63)$ & $\begin{array}{r}49,5(24- \\
78)\end{array}$ & $32(22-66)$ & $53(24-68)$ & $51(24-79)$ \\
\hline $\begin{array}{l}\text { Present } \\
n=53(43.8 \%)\end{array}$ & $36(20-71)$ & $33(12-64)$ & $41(26-63)$ & $42(24-64)$ & $41(24-62)$ & $51(25-74)$ & $35(20-64)$ & $57(24-64)$ & $49(24-77)$ \\
\hline $\mathbf{p}$ & 0,259 & 0,229 & 0,106 & 0,163 & 0,078 & 0,123 & 0,256 & 0,185 & 0,720 \\
\hline
\end{tabular}

SF: Short Form, PF: physical functioning, PR: physical role, BP: bodily pain, GH: general health, V: vitality, SF: social functioning, ER: emotional role, M: mental health, T.: transport, Transp.: transportation, H.S-U: High school or university

When the correlation between SF-36 results and ZCBI caregiver burden results was examined; Spearman correlation analysis showed moderate to strong negative correlation between ZCBI and all SF-36 results ( $\mathrm{p}<0.001$, r: -646 to -749 ). Additionally patient and caregiver ages represents positive correlation with zarit scores ( $\mathrm{p}<0.001, \mathrm{r}$ : 324 and $\mathrm{p}=0.002$, $\mathrm{r}: 275$ respectively) (table 3 ).

The distribution of ZCBI score by age is shown in Figure 1. With the increase in the age of the patient and the age of the caregiver, the ZCBI scores also increased. Roc curve analysis was performed on the results of the ZCBI caregiver burden questionnaire and the cut off age of 47 resulted in the sensitivity of $\% 69.57$ and the specificity as \% 65.52 (AUC $=0.671, \mathrm{P}=0.008$ ) (figure 2).
Table III: Spearman correlation analysis of Zarit Scores and SF36 scores and age

\begin{tabular}{|llr|}
\hline & & Zarit \\
\hline SF36 physical function & $\mathrm{r}$ &,- 694 \\
SF36 physical role & $\mathrm{p}$ & $<0,001$ \\
SF36 pain & $\mathrm{r}$ &,- 646 \\
& $\mathrm{p}$ & $<0,001$ \\
SF36 overall health & $\mathrm{r}$ &,- 705 \\
& $\mathrm{p}$ & $<\mathbf{0 , 0 0 1}$ \\
SF36 vitality & $\mathrm{r}$ &,- 716 \\
& $\mathrm{p}$ & $<0,001$ \\
SF36 social & $\mathrm{r}$ &,- 681 \\
& $\mathrm{p}$ & $<0,001$ \\
SF36 emotion & $\mathrm{r}$ &,- 718 \\
& $\mathrm{p}$ & $<0,001$ \\
SF36 mental health & $\mathrm{r}$ &,- 649 \\
& $\mathrm{p}$ & $<\mathbf{0 , 0 0 1}$ \\
Age & $\mathrm{r}$ &,- 739 \\
& $\mathrm{p}$ & $<0,001$ \\
Caregiver age & $\mathrm{r}$ &, 324 \\
& $\mathrm{p}$ & $<0,001$ \\
Sf36: short form 36. & $\mathrm{r}$ &, 275 \\
& $\mathrm{p}$ & $\mathbf{0 0 0 2}$ \\
\hline
\end{tabular}




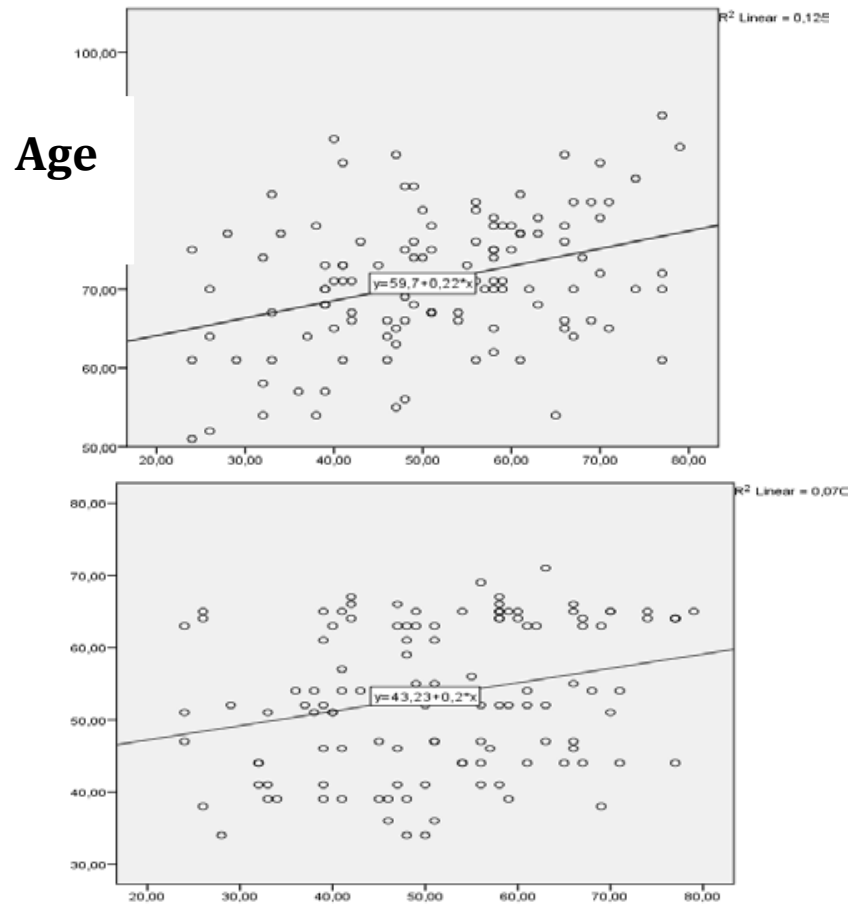

Zarit score

Figure 1. Distribution of Zarit Scores by age: patient and caregiver respectively

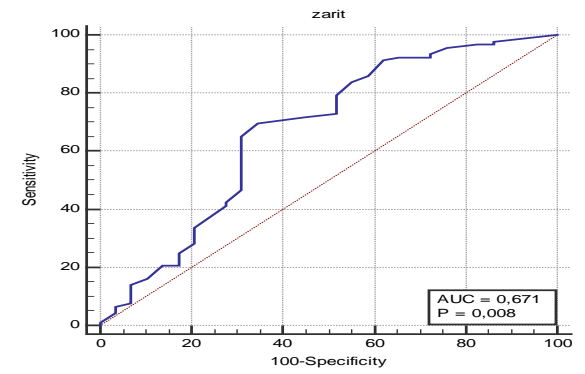

\begin{tabular}{|llllll|}
\hline $\begin{array}{l}\text { Cut } \\
\text { Off }\end{array}$ & $\begin{array}{l}\text { Sensitivity } \\
(95 \% \mathrm{CI})\end{array}$ & $\begin{array}{l}\text { Spesificity } \\
(95 \% \mathrm{CI})\end{array}$ & $\begin{array}{l}\text { AUC } \pm \text { se } \\
(95 \% \mathrm{CI})\end{array}$ & $\mathrm{p}$ \\
\hline $\begin{array}{l}\text { Zarit } \\
\text { scor } \\
\mathrm{e}\end{array}$ & $>47$ & 69,57 & 65,52 & 0,671 & $\mathbf{0 , 0 0}$ \\
$(59,1-78,7)$ & $(45,7-82,1)$ & $(0,579-0,753)$ & $\mathbf{8}$ \\
\hline
\end{tabular}

Figure 2. Zarit Score Sensitivity - Specificity Analysis

When the patients were evaluated as under 75 years old and over; Lower quality of life and higher caregiver burden were observed in all parameters except SF $36 \mathrm{GH}$ and M.

\section{DISCUSSION}

In our study, the caregiver burden and the patients' results of the SF-36 questionnaires in metastatic prostate cancer patients were examined in terms of the clinical and socioeconomic conditions of the patients. To our knowledge, this study is the first to comparatively evaluate the quality of life, disease-related clinical factors, caregiver characteristics and socioeconomic status in prostate cancer patients as a whole.

The presence of comorbidities, high ECOG score, the number of bone metastases and the presence of bone related complications were significant patient-related factors that negatively affected the survey results. In a study examining anxiety and quality of life in prostate cancer patients, it was revealed that the quality of life was negatively affected in patients with high degree of anxiety related to cancer ${ }^{19}$. In the quality of life assessment performed by Vieira et al. to assess pain in patients with bone metastasis; it was determined that most of the female patients had metastatic breast cancer and the male patients had metastatic prostate cancer. It was observed that male patients had to cope with more severe pain at an older age and their quality of life was found to be lower (Vieira C).

It was observed that the results of the questionnaire were negatively affected by the treatment-related factors, chemotherapy and palliative radiotherapy. Uemura et al. Showed that the use of new generation antiandrogens and an symptom control focused active patient follow-up procedures are associated with higher quality of life and lower care burden in bone metastatic prostate cancer patients ${ }^{20}$. Since our study was cross sectional, whether the patients had received active chemotherapy in the last 6 months or less (including the time while data was collected) was evaluated. Consequently, higher caregiver burden and lower quality of life can be observed in cases experiencing chemotherapy side effects. However, it is clear that chemotherapy improves the quality of life in eligible patients in castration resistant metastatic prostate cancer ${ }^{21}$. There is still controversy about the 
timing of chemotherapy with new generation antiandrogens in sequential treatment algorithms. This is not a focus of our study.

Among socioeconomic factors, it has been observed that the obligation to use public transport, being a resident in the village and being a tenant negatively affected the survey results at various levels. Previous studies described that the caregivers of patients with chronic diseases are exposed to financial, psychological and physical burden $3,4,6$. Caregivers might have to quit working, reduce working time, and loss income due to hospital visits or hospitalizations ${ }^{22}$. Soen et al. evaluated the caregiver burden of families of patients with osteoporotic fractures. Sixty-one percent of caregiver family members developed financial problems such as not being able to continue their profession as before and they experienced economic stress ${ }^{23}$.

Although there was no strong relationship between caregiver-related factors in our study, the condition that the caregiver was someone other than a spouse, sibling or child could be interpreted as a negative factor. Caregivers without social support can feel isolated and alone in the care giving process. It could have resulted in higher levels of stress and family conflicts, further exacerbating this condition ${ }^{5}$. Faronbi et al. reported that religion was the most used strategy for coping ability in caregivers of adult patients ${ }^{24}$. Rahmani et al. described that caregivers of schizophrenia patients had severe burden to all family3 ${ }^{3}$

In order to make a statistical verification in our study, when the factors affecting the ZCBI questionnaire results were evaluated, it was observed that there was a positive correlation with ECOG, caregiver age, public transportation obligation and various SF-36 results. In addition, a significant negative correlation between the ZCBI questionnaire and SF-36 results has been demonstrated. Srivastava et al. reported a negative correlation among QoL and burden in caregivers of dementia patients ${ }^{25}$. Similarly; Jafari et al. described a negative correlation between QoL and burden in caregivers of hemodialysis patients ${ }^{26}$.

Our study looks to provide critical insight into how to relieve the economic burden laid upon advanced cancer patients, their caregivers and the healthcare system. There are few studies that shed light on the more systematic transfer of health budget resources to the needs of patients and their families. In their study in which they evaluated 832 bone metastatic patients diagnosed with castration resistant prostate cancer, McKay et al. detected symptomatic skeletal events in 207 patients during the cohort. The relationship between bone events, which mostly result in emergency admission with poor quality of life, care burden and economic burden has been shown. They highlighted the importance of palliative approaches and the rational use of cancer therapies ${ }^{27}$. In their studies addressing the subject in a similar context, Uemura et al. showed that the symptomatic bone event is associated with increased healthcare costs and analgesic use $\mathrm{e}^{20}$. At this point, the support needs of routine follow-up patients can be measured with systematic measurement methods. We believe that a more accurate management of health economics' orientation towards patient service will be possible with this method. The method in which ZCBI caregiver burden and SF36 are evaluated together has proven to be useful for measuring the burden of the family in coping with the disease. Healthcare services in our country are primarily provided by the state. We think that it is critical to direct both the state and private health services correctly. Considering the fact that patient demands are effective in addition to objective medical examination results, active use and monitoring of scales in routine polyclinic functioning, as we did in this study, may be beneficial. It can be 
rational and efficient to recruit employees who are specialized in this matter.

Patients who took second-generation androgen pathway inhibitors were not included in our study. Because the majority of these patients are in the group that received multiple-stage treatment. Since the rate of patients with severely low performance status has increased and there may be inconvenience in terms of objective evaluation, these patients were not included in our study for the purpose of homogenization.

The most important limitation of our study is that it is cross-sectional. A prospective study following a long course of treatment could more objectively evaluate the effect of therapeutic factors. In addition, the fact that it is a single center study can be considered as a limiting factor in terms of not being able to examine different geographical and cultural habitats.

In conclusion; it was found that the quality of life in patients with metastatic prostate cancer was directly related to caregiver burden. Factors such as patient comorbidities, bone metastasis, ECOG score, development of bone incident and associated palliative radiotherapy requirement, presence of visceral metastasis, being under active chemotherapy, low socioeconomic status, and the condition of caregiver not being a spouse, sibling or extra-foster relatives negatively affect both the quality of life and caregiver burden. If a social assistance system can be implemented in which socioeconomic conditions and patient-related factors can be monitored more systematically, objective and cost-effective benefits related to the care of these patients and the maintenance of their quality of life can be achieved.

Ethics Committee Approval: This study was approved by The Gazi Yaşargil Training and Research Hospital Ethics Board (25.09.2020/564) and was applied in accordance of the Declaration of Helsinki.
Conflict of Interest: No conflict of interest was declared by the authors.

Financial Disclosure: No financial disclosure was declared by the authors.

\section{REFERENCES}

1. Burckhardt CS, Woods SL, Schultz AA, Ziebarth DM. Quality of life of adults with chronic illness: A psychometric study. Res Nurs Health. 1989; 12: $347-54$.

2. Buyan N, Türkmen MA, Bilge I, et al. Quality of life in children with chronic kidney disease (with child and parent assessments). Pediatr Nephrol. 2010; 25: 1487-96.

3. Rahmani F, Ranjbar F, Hosseinzadeh M, et al. Coping strategies of family caregivers of patients with schizophrenia in Iran: A cross-sectional survey. Int J Nurs Sci. 2019; 6: 148-53.

4. Mora-Castañeda B, Márquez-González M, Fernández-Liria A, de la Espriella R, Torres N, Arenas Borrero Á Clinical and demographic variables associated coping and the burden of caregivers of schizophrenia patients. Rev Colomb Psiquiatr. 2018; 47: 13-20.

5. Parekh NK, Shah S, McMaster K, et al. Effects of caregiver burden on quality of life and coping strategies utilized by caregivers of adult patients with inflammatory bowel disease. Ann Gastroenterol. 2017; 30: 89-95.

6. Cooper C, Katona C, Orrell M, Livingston G. Coping strategies, anxiety and depression in caregivers of people with Alzheimer's disease. Int J Geriatr Psychiatry. 2008; 23: 929-36.

7. Johansen S, Cvancarova M, Ruland C. The effect of cancer patients' and their family caregivers' physical and emotional symptoms on caregiver burden. Cancer Nurs. 2018; 41: 91-99.

8. Semere W, Althouse AD, Rosland AM, et al. Poor patient health is associated with higher caregiver burden for older adults with advanced cancer. J Geriatr Oncol. 2021; 5: 771-8.

9. Crowder SL, Najam N, Sarma KP, Fiese BH, Arthur AE. Quality of life, coping strategies, and supportive care needs in head and neck cancer survivors: a 
qualitative study. Support Care Cancer. 2021; 8: 4349-56.

10. Guan T, Santacroce SJ, Chen DG, Song L. Illness uncertainty, coping, and quality of life among patients with prostate cancer. Psychooncology. 2020; 29: 1019-25.

11. Wang L, Paller CJ, Hong H, et al. Comparison of Systemic Treatments for Metastatic CastrationSensitive Prostate Cancer: A Systematic Review and Network Meta-analysis. JAMA Oncol. 2021; 3: 41220.

12. Palumbo C, Bruni A, Antonelli A, et al.; Pros-IT CNR Study Group. Health-related quality of life 24month after prostate cancer diagnosis: an update from the Pros-IT CNR prospective observational study. Minerva Urol Nefrol. 2021;1. Epub ahead of print. PMID: 33439570.

13. American Diabetes Association. 11. Older Adults. Diabetes Care. 2017; 1: 99-104

14. McHorney CA, Ware Jr JE, Raczek AE. The MOS 36-Item Short-Form Health Survey (SF-36): II. Psychometric and clinical tests of validity in measuring physical and mental health constructs. Med Care 1993; 31: 247-63.

15. Zarit SH, Reever KE, Bach-Peterson J. Relatives of the impaired elderly: correlates of feeling of burden. Gerontologist 1980; 20: 649-55.

16. Özlü A, Yıldız M, Aker T. A reliability and validity study on the ZCBI caregiver burden scale. Archives of Neuropsychiatry 2009; 46: 38-42

17. Heidenreich A, Pfister D, Porres D. Cytoreductive radical prostatectomy in patients with prostate cancer and low volume skeletal metastases: results of a feasibility and case-control study. J Urol. 2015; 193: 832-8.

18. Tosoian JJ, Gorin MA, Ross AE, et al. Oligometastatic prostate cancer: definitions, clinical outcomes, and treatment considerations. Nat Rev Urol. 2017; 14: 15-25.
19. Erim DO, Bennett AV, Gaynes BN, et al. Associations between prostate cancer-related anxiety and health-related quality of life. Cancer Med 2020; 9: 4467-73.

20. Uemura H, DiBonaventura M, Wang E, et al. The treatment patterns of castration-resistant prostate cancer in Japan, including symptomatic skeletal events and associated treatment and healthcare resource use. Expert Rev Pharmacoecon Outcomes Res 2017; 17: 511-7.

21. Ferro M, Lucarelli G, Crocetto F, et al. First-line systemic therapy for metastatic castration-sensitive prostate cancer: An updated systematic review with novel findings. Crit Rev Oncol Hematol. 2021; 157: 103-198.

22. Brust JD, Leonard BJ, Sielaff BH. Maternal time and the care of disabled children. Public Health Nurs. 1992; 9: 177-184.

23. Soen S, Usuba K, Crawford B, Adachi K. Family caregiver burden of patients with osteoporotic fracture in Japan. J Bone Miner Metab. 2021; 4: 61222.

24. Faronbi JO. Correlate of burden and coping ability of caregivers of older adults with chronic illness in Nigeria. Scand J Caring Sci. 2018; 32: 128896.

25. Srivastava G, Tripathi RK, Tiwari SC, Singh B, Tripathi SM. Caregiver Burden and Quality of Life of Key Caregivers of Patients with Dementia. Indian J Psychol Med. 2016; 38: 133-6.

26. Jafari H, Ebrahimi A, Aghaei A, Khatony A (2018) The relationship between care burden and quality of life in caregivers of hemodialysis patients. BMC Nephrol 19: 321.

27. McKay R, Haider B, Duh MS, et al. Impact of symptomatic skeletal events on health-care resource utilization and quality of life among patients with castration-resistant prostate cancer and bone metastases. Prostate Cancer Prostatic Dis. 2017; 20: 276-82. 